University of Nebraska - Lincoln

DigitalCommons@University of Nebraska - Lincoln

\title{
Olanzapine and risperidone disrupt conditioned avoidance responding by selectively weakening motivational salience of conditioned stimulus: Further evidence
}

\author{
Chen Zhang \\ Shanghai Mental Health Center, Shanghai Jiaotong University School of Medicine \\ Yiru Fang \\ Shanghai Mental Health Center, Shanghai Jiaotong University School of Medicine \\ Ming Li \\ University of Nebraska-Lincoln, mli2@unl.edu
}

Follow this and additional works at: https://digitalcommons.unl.edu/psychfacpub

Part of the Psychiatry and Psychology Commons

Zhang, Chen; Fang, Yiru; and Li, Ming, "Olanzapine and risperidone disrupt conditioned avoidance responding by selectively weakening motivational salience of conditioned stimulus: Further evidence" (2011). Faculty Publications, Department of Psychology. 565.

https://digitalcommons.unl.edu/psychfacpub/565

This Article is brought to you for free and open access by the Psychology, Department of at DigitalCommons@University of Nebraska - Lincoln. It has been accepted for inclusion in Faculty Publications, Department of Psychology by an authorized administrator of DigitalCommons@University of Nebraska - Lincoln. 


\title{
Olanzapine and risperidone disrupt conditioned avoidance responding by selectively weakening motivational salience of conditioned stimulus: Further evidence
}

\author{
Chen Zhang, ${ }^{1,2}$ Yiru Fang, ${ }^{1}$ and Ming Li ${ }^{2}$ \\ 1. Shanghai Mental Health Center, Shanghai Jiaotong University School of Medicine, \\ 600 Wan Ping Nan Road, Shanghai 200030, PR China \\ 2. Department of Psychology, University of Nebraska-Lincoln, Lincoln, NE 68588-0308, USA \\ Corresponding author - M. Li, Department of Psychology, 238 Burnett Hall, University of Nebraska-Lincoln, \\ Lincoln, NE 68588-0308, USA; tel 402 472-3144, email mli2@unl.edu
}

\begin{abstract}
Suppression of conditioned avoidance response is a preclinical behavioral index of antipsychotic activity. Previous work shows that olanzapine and risperidone disrupt avoidance response elicited by a less salient conditioned stimulus (CS2) to a greater extent than avoidance elicited by a more salient stimulus (CS1), suggesting that antipsychotic drugs may have a weakening action on motivational salience of stimuli. In the present study, we further examined this mechanism of antipsychotic action, focusing on the possible impact of baseline difference of CS1 and CS2 response rates on the avoidance-disruptive effect of olanzapine and risperidone. Rats were first trained to acquire avoidance responding in a procedure in which the number of CS2 trials (i.e. 20) was twice the number of CS1 trials (i.e. 10), but the percentage of CS2-shock pairing was set at 25\% lower (15 trials out of 20) than the percentage of CS1shock pairing (20 trials out of 20$)$. They were then tested daily under olanzapine $(0.5$ and $1.0 \mathrm{mg} / \mathrm{kg}$, sc) or risperidone $(0.33$ and $1.0 \mathrm{mg} / \mathrm{kg}$, sc) for 5 consecutive days. Repeated olanzapine and risperidone treatment dose-dependently disrupted avoidance responding to both CS1 and CS2. Both drugs at the high dose disrupted the CS2 avoidance to a greater extent than the CS1 avoidance. In the final challenge test, rats previously treated with olanzapine were tested under risperidone $(0.33 \mathrm{mg} / \mathrm{kg})$, whereas rats previously treated with risperidone were tested under olanzapine $(0.5 \mathrm{mg} / \mathrm{kg})$. Results show that rats previously treated with risperidone $1.0 \mathrm{mg} / \mathrm{kg}$ group made significantly fewer avoidance responses than the vehicles under olanzapine at $0.5 \mathrm{mg} / \mathrm{kg}$. These findings confirm that olanzapine and risperidone disrupt avoidance response primarily by selectively attenuating the motivational salience of the CS. The present study also suggests that there is a generality of antipsychotic drug experience that is mediated by a shared interoceptive drug state mechanism.
\end{abstract}

Keywords: olanzapine, risperidone, motivational salience, conditioned avoidance response, behavioral sensitization

\section{Introduction}

The rat conditioned avoidance response (CAR) model is a commonly used preclinical test for antipsychotic activity (Arnt, 1982). All currently used antipsychotics at clinically relevant doses selectively disrupt avoidance response to a conditioned stimulus (CS, e.g. white noise) without altering escape response to an unconditioned stimulus (US, e.g. footshock). This feature has been effectively used to identify potential antipsychotic drugs, to differentiate antipsychotic drugs from other classes of psychotropic drugs, and to predict the clinical potency of antipsychotic drugs and (Arnt, 1982; Bignami, 1978; Cook and Davidson, 1978; Janssen et al., 1965; Kuribara and Tadokoro, 1981; Shannon et al., 1999; van der Heyden and Bradford, 1988; Wadenberg and Hicks, 1999). Therefore, understanding the nature of antipsychotic-induced avoidance disruption may shed light on the behavioral and neurobiological mechanisms of antipsychotic action in the treatment of psychosis.
In recent years, we have reported several studies that examined the behavioral mechanisms of antipsychotic effect in the CAR model (Li et al., 2004, 2007, 2009a, 2009b, 2010; Mead and $\mathrm{Li}, 2010)$. Our overall results suggest that typical and atypical antipsychotic drugs disrupt avoidance via two separate mechanisms: (1) attenuating the motivational salience of the CS and (2) providing an interoceptive drug cue that allows the decreased salience of the CS to be maintained over time. The attenuation action on motivational salience of the CS refers to the weakening effect of antipsychotic treatment on the ability of the CS to instigate an active motor response from an organism. One interesting finding in support of this action comes from our recent study (Li et al., 2009b), in which we examined olanzapine and risperidone in a modified avoidance conditioning procedure involving two types of CS (CS1: a white noise and CS2: a pure tone). These two CSs varied in their salience and ability to predict the occurrence of the US (the CS1 was paired with the US in every trial, whereas the CS2 was paired with the US in only half of the trials). We found 
that both drugs had a stronger disruptive effect on a less salient CS2-elicited avoidance than a highly salient CS1-elicited avoidance. Although this result is consistent with our motivational salience attenuation hypothesis, there are alternative explanations. For example, this differential action may result from the different baseline levels of CS1 and CS2 avoidance. In the previous study ( $\mathrm{Li}$ et al., 2009b), the mean CS1 avoidance (about $95 \%$ of 20 trials) was significantly higher than the mean CS2 avoidance ( $50 \%$ of 10 trials) on the pre-drug day. This difference in avoidance responding rate at baseline could have contributed to the greater disruptive effects of antipsychotic drugs on CS2 avoidance, independent of motivational salience of CS1 and CS2. There are many published examples which demonstrate that differences in scheduling of events and rate of behavioral responding can determine the direction and magnitude of the behavioral effects of drugs (Barrett, 2002; Barrett et al., 2008; Dews, 1976; McMillan and Katz, 2002; Spealman et al., 1983). Thus, the primary purpose of the present study was to further investigate the motivational salience attenuation action of olanzapine and risperidone and to distinguish between these two alternative interpretations ("motivational salience" versus "different baseline response"). In doing so, we created a new CAR procedure in which the number of CS2 trials (i.e. 20) was twice the number of CS1 trials (i.e. 10), but the percentage of CS2-US (shock) pairing was set at $25 \%$ (15 trials out of 20) lower than the percentage of CS1-US shock pairing (20 trials out of 20). Under this condition, the CS1 was still more salient than the CS2, but the baseline CS2 avoidances were higher than CS1 avoidances. If olanzapine and risperidone were to decrease CS2 avoidance to a greater extent than CS1 avoidance, it would provide additional evidence in support of the motivational salience interpretation.

The secondary purpose was to examine the interoceptive drug state mechanism. Our previous work shows that antipsychotic drugs haloperidol and olanzapine share a common "antipsychotic" interoceptive drug state, as evidenced by the findings that rats previously treated with haloperidol (or olanzapine) showed a stronger response to the avoidance-disruptive effect of olanzapine (or haloperidol) than drug-naïve rats (Li et al., 2007; Mead and Li, 2010). The present study examined the possible shared interoceptive drug state between olanzapine and risperidone and also investigated whether prior experience with one drug (e.g. olanzapine) could be transferrable to another drug (e.g. risperidone).

\section{Materials and methods}

\subsection{Animals}

Male Sprague-Dawley rats (226-250 g upon arrival, Charles River, Portage, MI) were housed two per cage, in $48.3 \mathrm{~cm} \times 26.7 \mathrm{~cm} \times 20.3 \mathrm{~cm}$ transparent polycarbonate cages under 12-h light/dark conditions (light on between 6:30 am and $6: 30 \mathrm{pm}$ ). Room temperature was maintained at $22 \pm 1{ }^{\circ} \mathrm{C}$ with an averaged humidity around $45 \%$. Food and water were available ad libitum. Animals were allowed at least one week of habituation to the animal facility before being used in experiments. All procedures were approved by the Institutional Animal Care and Use Committee at the University of Nebraska-Lincoln.

\subsection{Two-way avoidance conditioning apparatus}

Eight identical two-way shuttle boxes custom designed and manufactured by Med Associates (St. Albans, VT) were used. Each box was housed in a ventilated, sound-insulated isolation cubicle $(96.52 \mathrm{~cm} \mathrm{~W} \times 35.56 \mathrm{~cm} \mathrm{D} \times 63.5 \mathrm{~cm} \mathrm{H})$. Each box was $64 \mathrm{~cm}$ long, $30 \mathrm{~cm}$ high (from grid floor), and $24 \mathrm{~cm}$ wide, and was divided into two equal-sized compartments by a partition with an arch style doorway ( $15 \mathrm{~cm}$ high $\times 9 \mathrm{~cm}$ wide at base). A barrier ( $4 \mathrm{~cm}$ high) was placed between the two compartments, so the rats had to jump from one compartment to the other. The grid floor consisted of 40 stainless-steel rods with a diameter of $0.48 \mathrm{~cm}$, spaced $1.6 \mathrm{~cm}$ apart center to center, through which a scrambled footshock (US, $0.8 \mathrm{~mA}$, maximum duration: $5 \mathrm{~s}$ ) was delivered by a constant current shock generator (Model ENV-410B) and scrambler (Model ENV-412). Illumination was provided by two houselights mounted at the top of each compartment. The CSs (either a $76 \mathrm{~dB}$ white noise CS1 or an $85 \mathrm{~dB}$ $2800 \mathrm{~Hz}$ pure tone CS2) were produced by a speaker (ENV 224 AMX) mounted on the ceiling of the cubicle, centered above the shuttle box. Background noise (approximately $74 \mathrm{~dB}$ ) was provided by a ventilation fan affixed at the top corner of each isolation cubicle. All training and testing procedures were controlled by Med Associates programs running on a computer.

\subsection{Drugs}

Olanzapine (OLZ) and risperidone (RIS) (gifts from NIMH Chemical Synthesis and Drug Supply Program) were dissolved in $1.0 \%$ glacial acetic acid in distilled water. We chose olanzapine at 0.5 and $1.0 \mathrm{mg} / \mathrm{kg}$ and risperidone at 0.33 and $1.0 \mathrm{mg} / \mathrm{kg}$ because these doses are effective in producing a robust dose-dependent disruption of avoidance responding ( $\mathrm{Li}$ et al., 2007; Li et al., 2009b), and both drugs at these doses give rise to clinically comparable levels of striatal D2 occupancy (60-70\%) (Kapur et al., 2003).

\subsection{Procedure}

Forty-eight male Sprague-Dawley rats (226-250 g upon arrival, Charles River, Potage, MI, USA) were used. After 2 days of shuttle box habituation, all rats were trained daily over ten consecutive days in the modified two-way avoidance conditioning task for a total of 10 sessions. Each training session consisted of 30 trials. Ten trials (CS1 trials) used a $10 \mathrm{~s} 76 \mathrm{~dB}$ white noise as the CS with its termination immediately followed by a shock ( $0.8 \mathrm{~mA}$, maximum duration: $5 \mathrm{~s})$ if the rats did not make an avoidance response. The remaining 20 trials (CS2 trials) used a pure tone $(10 \mathrm{~s}, 2800 \mathrm{kHz}, 85 \mathrm{~dB})$ as the CS. In 15 CS2 trials, the CS2 was followed by the shock if the rat failed to respond to the CS2; whereas, in the remaining 5 trials, it was not. The 10 CS1 trials were randomly intermixed with the 20 CS2 trials. During each trial, if a subject moved from one compartment into the other within the 10s of CS presentation, that CS was immediately terminated, the shock was prevented and this shuttling response was recorded as avoidance (termed CS1 avoidance or CS2 avoidance). If the rat remained in the same compartment for more than $10 \mathrm{~s}$ and made a crossing upon receiving the footshock, this response was recorded as escape. If the rat did not respond during the entire $5 \mathrm{~s}$ presentation of the shock, the trial was terminated and escape failure was recorded. At the end of the training session period, 39 rats had reached training criterion ( $\geq 7$ CS1 avoidances and at least one CS2 avoidance in the last two training sessions). They were first matched on avoidance performance on the last training day to create blocks of rats ( $n=4-5$ rats/block) that were approximately equal in performance. Within each block, they were then randomly assigned to one of five groups: vehicle control (VEH, $n=11)$, RIS $0.33 \mathrm{mg} / \mathrm{kg}(n=7)$, RIS $1.0 \mathrm{mg} /$ $\mathrm{kg}(n=7)$, OLZ $0.5 \mathrm{mg} / \mathrm{kg}(n=7)$ and OLZ $1.0 \mathrm{mg} / \mathrm{kg}(n=7)$. They were then subjected to 5 consecutive days of repeated VEH, RIS, or OLZ testing. On each test day, rats were injected with VEH (sterile water), RIS (0.33 or $1.0 \mathrm{mg} / \mathrm{kg}$ ) or OLZ $(0.5$ or $1.0 \mathrm{mg} / \mathrm{kg}$ ) subcutaneously (sc) $1 \mathrm{~h}$ before being placed in the apparatus. One day after the last drug test, all rats were retrained drug-free in two sessions to bring their avoidance back to the pre-drug level. A final drug challenge test was conducted $24 \mathrm{~h}$ after the last retraining session to assess the pos- 
sible shared interoceptive drug state and interchangeability between OLZ and RIS treatment sensitization (Li et al., 2007; Mead and Li, 2010). During the test, rats in the VEH group were randomly assigned into two subgroups and injected with OLZ $0.5 \mathrm{mg} / \mathrm{kg}(n=5)$ or RIS $0.33 \mathrm{mg} / \mathrm{kg}(n=6)$. Rats in the two OLZ groups were all injected with RIS $0.33 \mathrm{mg} / \mathrm{kg}$, and rats in the two RIS groups were injected with OLZ $0.5 \mathrm{mg} / \mathrm{kg}$. These challenge doses were chosen because they have been demonstrated to be effective doses in revealing prior antipsychotic treatment effect (Li et al., 2010; Mead and Li, 2010). The same CAR procedure used during training was used throughout the drug testing phase.

\subsection{Statistical analysis}

Avoidance response data from the training sessions were expressed as the mean number of avoidance responses and percent CS1 (number of avoidances divided by 10) and CS2 (number of avoidances divided by 20) avoidance. As done in our previous work (Li et al., 2009b), avoidance response data from the drug test sessions were expressed as the percent avoidance on each drug test session relative to each rat's own avoidances made on the last training (10th) day ( $\%$ avoidance $=$ number of avoidance in any drug session / number of avoidance on the 10th training day). This measure better accounted for the differences in the baseline number of CS1 (max: 10 trials) and CS2 (max: 20) trials as well as individual differences at baseline. Data from the final challenge test was expressed as mean number of avoidance responses. Data from the five drug test sessions were analyzed using a two-way mixed-design analysis of variance (ANOVA) with the between-subjects factor being drug treatment and the within-subjects factors being test session and type of trials (i.e. CS1 or CS2 avoidance). Individual one-way ANOVAs were used to detect group differences on CS1 and CS2 avoidances separately on each drug test session and two retraining sessions. Data on the final olanzapine and risperidone challenge sessions were analyzed separately using one-way ANOVAs. CS1 and CS2 avoidances were also examined separately. A conventional two-tailed level of significance at the $5 \%$ level was required.

\section{Results}

\subsection{CAR training phase: CS1 avoidance was acquired faster than CS2 avoidance}

Throughout the 10 training sessions, avoidance response to the CS1 and CS2 showed a progressive increase across sessions (Figure 1). A two-way mixed-design ANOVA on the number of avoidances indicated a main effect of session, $F(9$, $342)=80.877, p<0.001$, type, $F(1,38)=90.138, p<0.001$, and a significant session $\times$ type interaction, $F(9,342)=48.433, p<0.001$. A similar analysis on the percent avoidance revealed a similar main effect of session and type, but no session $\times$ type interaction, $F(9,342)=1.591, p=0.116$. Inspection of the training data indicated that even the daily mean numbers of CS2 avoidance were higher than CS1 avoidance (Figure 1A), the mean percent CS1 avoidance was higher than CS2 avoidance (Figure 1B), confirming that the CS2 stimulus was indeed less salient in instigating avoidance response than the CS1. This result was expected from the experimental design in which only $75 \%$ of the CS2 trials (15 out of 20) were followed by shock, whereas all 10 CS1 trials were followed by shock if the rats failed to respond.

\subsection{Drug testing phase: olanzapine and risperidone disrupted CS2 avoidance to a greater extent than CS2 avoidance}

As can be seen in Figure 2, across the 5 drug test sessions, the VEH-treated rats maintained their levels of CS1 and
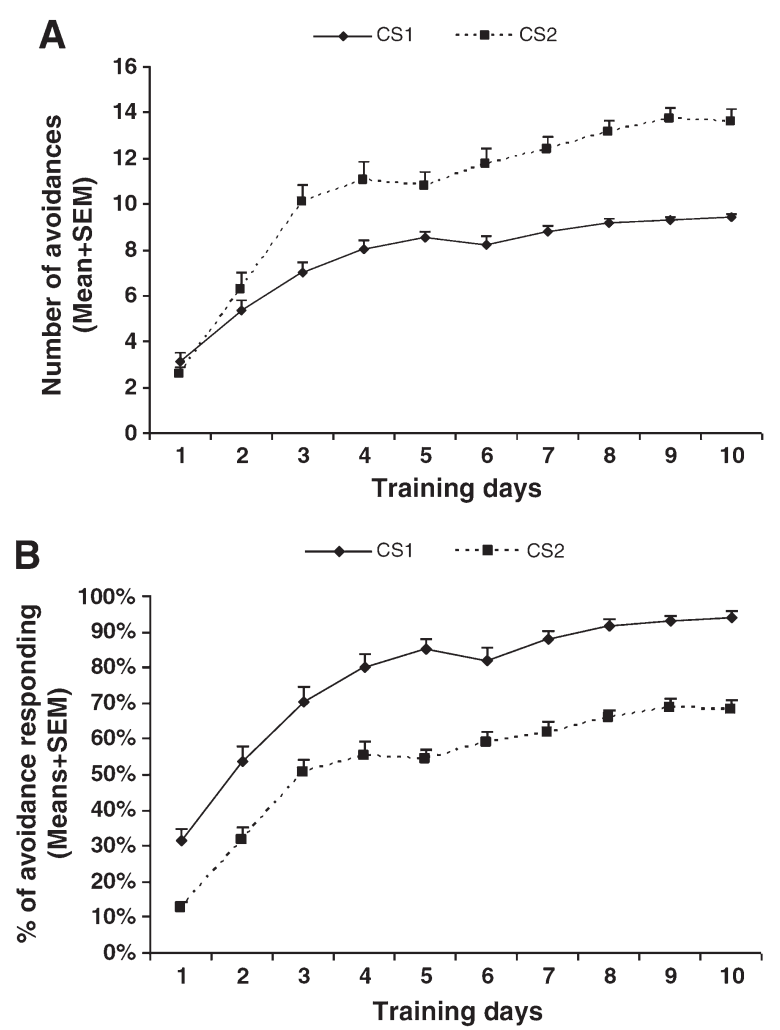

Figure 1. Acquisition of CS1 and CS2 avoidance response across the 10 training sessions. Each point represents either mean number of avoidance responses + SEM (A) or mean avoidance percent + SEM (B). The number of CS2 avoidance was higher than CS1 avoidance, but the percent CS1 avoidance was higher than the CS2 avoidane.

CS2 avoidance throughout the sessions, whereas the RIS and OLZ rats showed a dose-dependent and progressive decline in both CS1 and CS2 avoidance responding. A two-way mixed-design ANOVA revealed a main effect of drug treatment, $F(4,34)=56.089, p<0.001$, test session, $F(4,136)=6.050$, $p<0.001$, and trial type, $F(1,34)=9.923, p=0.003$. The interactions between drug treatment and test session, $F(16,136)=1.993$, $p=0.018$, and between drug treatment and trial type, $F(4$, $34)=2.791, p=0.042$ were also significant, suggesting that OLZ and RIS had a differential effect on different types of avoidance trials across the drug test sessions.

For the CS1 trials, one-way ANOVAs involving all the groups revealed a main group effect on every day, $F(4$, $34)=26.591-35.484, p s<0.001$. Tukey post hoc tests showed that the two high-dose groups (i.e. OLZ $1.0 \mathrm{mg} / \mathrm{kg}$ and RIS $1.0 \mathrm{mg} / \mathrm{kg}$ ) differed significantly from the vehicle group on every drug day, $p \mathrm{~s}<0.011$. The RIS $0.33 \mathrm{mg} / \mathrm{kg}$ group differed from the VEH group from day 2 to day 5, ps $<0.035$, whereas the OLZ $0.5 \mathrm{mg} / \mathrm{kg}$ group did not differ from the VEH group on any of the drug test days, $p s>0.184$. The RIS $1.0 \mathrm{mg} / \mathrm{kg}$ group had a significantly lower avoidance response among the four drug groups. It differed significantly from the other groups on days 1, 2 and $3, p s<0.001$, and from the two lowdose groups on days 4 and 5, $p \mathrm{~s}<0.001$. In addition, the OLZ $1.0 \mathrm{mg} / \mathrm{kg}$ group also differed significantly from the OLZ $0.5 \mathrm{mg} / \mathrm{kg}$ group on the last four test days, $p \mathrm{~s}<0.031$, indicating a dose-dependent effect.

For the CS2 trials, one-way ANOVAs involving all the groups revealed a main group effect on every day, $F$ (4, $34)=16.261-32.123, p s<0.001$. Tukey post hoc tests showed that all drug groups except the OLZ $0.5 \mathrm{mg} / \mathrm{kg}$ group differed significantly from the vehicle group on every drug day, $p s<0.017$. Once again, the RIS $1.0 \mathrm{mg} / \mathrm{kg}$ group had the greatest effect among the four drug groups. It differed significantly from all 

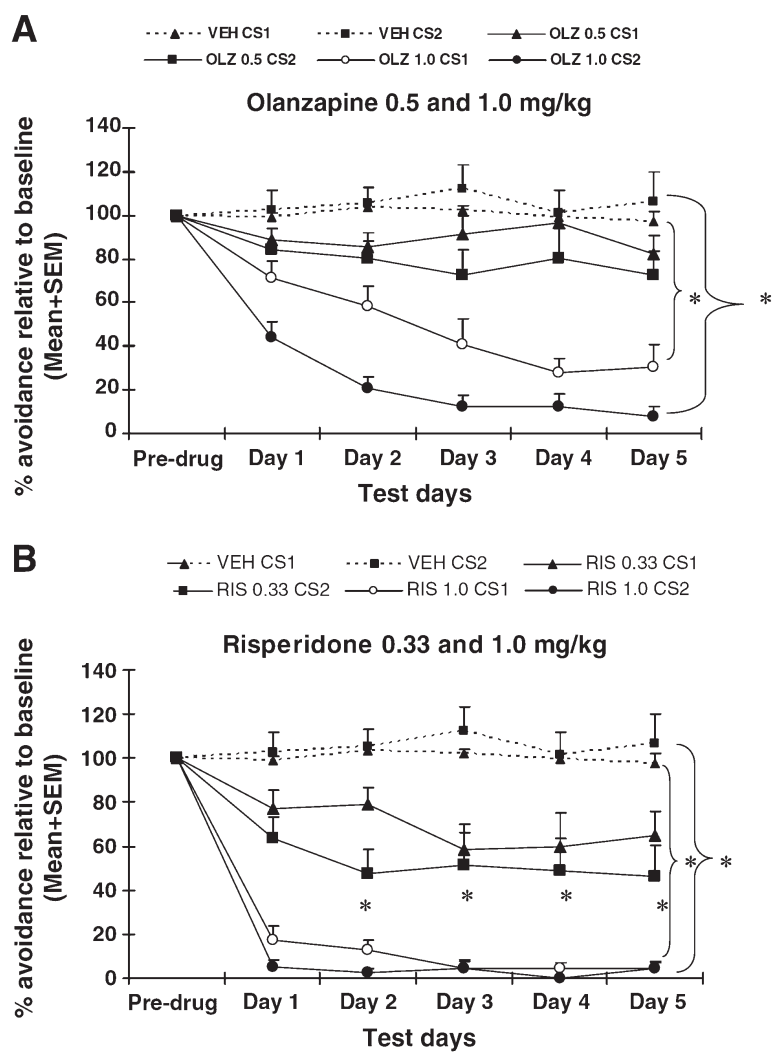

Figure 2. Effect of repeated olanzapine (A) or risperidone (B) treatment on CS1 and CS2 avoidance responding. Each point represents mean avoidance percent + SEM relative to the last (10th) training day. The avoidance data from the vehicle group were plotted in both figures for easy comparison. Olanzapine $(0.5$ and $1.0 \mathrm{mg} / \mathrm{kg}$, s.c., $-60 \mathrm{~min})$ and risperidone $(0.33$ and $1.0 \mathrm{mg} / \mathrm{kg}$, s.c., $-60 \mathrm{~min})$ dose-dependently disrupted avoidance response to both CS1 and CS2, and the disruption was progressively enhanced with repeated drug administration across the 5 daily test sessions. Olanzapine and risperidone had a stronger disruptive effect on CS2 avoidance than CS1 avoidance. " $*$ " indicates a significant difference $(p<0.05)$ between the antipsychotic group and the vehicle group.

other groups on day 1 , $p$ s $<0.040$, and from the two low-dose groups on days 2,3 and 4 , $p$ s $<0.045$, and also differed significantly from the OLZ $0.5 \mathrm{mg} / \mathrm{kg}$ group on day $5, p=0.003$. In addition, the OLZ $1.0 \mathrm{mg} / \mathrm{kg}$ group differed significantly from the OLZ $0.5 \mathrm{mg} / \mathrm{kg}$ group on every test day, $p \mathrm{~s}<0.028$.

\subsection{Final drug challenge test: number of avoidances}

During the two retraining sessions, both RIS and OLZ treated rats recovered quickly (data not shown). One-way ANOVAs on CS1 and CS2 avoidances on both sessions did not found any significant group difference, $F(4,34)=0.531-1.528$, ps $>0.215$. The final challenge test was designed to examine the similarity between the olanzapine and risperidone-induced drug state, and determine to what extent prior olanzapine or risperidone treatment potentiated later response to risperidone or olanzapine, respectively. As can be seen in Figure 3 , under the RIS $0.33 \mathrm{mg} / \mathrm{kg}$ challenge condition, all three groups had comparable levels of CS1 and CS2 avoidance responding. One-way ANOVA revealed no significant group effect for CS1 avoidance, $F(2,17)=0.037, p=0.964$ and CS2 avoidance, $F(2,17)=0.665, p=0.527$.

In contrast, under the OLZ $0.50 \mathrm{mg} / \mathrm{kg}$ challenge condition, rats that were previously treated with RIS $1.0 \mathrm{mg} / \mathrm{kg}$ made significantly fewer avoidance responses than rats that were previously treated with vehicle. One-way ANOVA revealed

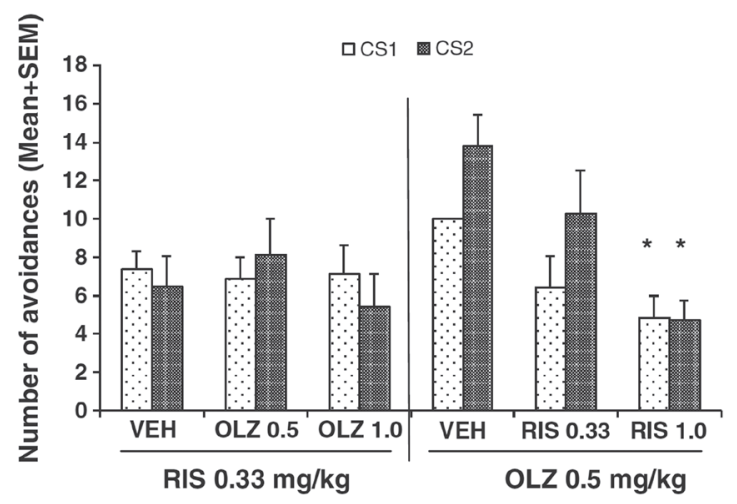

Figure 3. Effects of prior olanzapine or risperidone treatment on subsequent avoidance response to a challenge test of risperidone or olanzapine. Rats that were previously treated with risperidone ( 0.33 or $1.0 \mathrm{mg} / \mathrm{kg})$, olanzapine $(0.5$ and $1.0 \mathrm{mg} / \mathrm{kg}$ ) or vehicle during the drug testing phase were injected with olanzapine $(0.5 \mathrm{mg} / \mathrm{kg}$, s.c., $-60 \mathrm{~min})$ or risperidone $(0.33 \mathrm{mg} / \mathrm{kg}$, s.c., $-60 \mathrm{~min})$. Olanzapine, but not risperidone challenge, caused a greater decrease in avoidance responding in rats that were previously treated with risperidone $(1.0 \mathrm{mg} / \mathrm{kg})$. Each point represents either mean number of avoidance responses + SEM. "*" indicates a significant difference $(p<0.05)$ between the antipsychotic group and the vehicle group.

a main group effect for the CS1 avoidance, $F(2,16)=3.867$, $p=0.043$, and the CS2 avoidance, $F(2,16)=6.759, p=0.007$. Post hoc Tukey tests under each challenge condition showed that the RIS $1.0 \mathrm{mg} / \mathrm{kg}$ group differed significantly from the vehicle on these measures (all $p s<0.037$ ). No other group difference was found.

\section{Discussion}

Consistent with our previous study (Li, He, 2009b), the present study demonstrated that repeated olanzapine or risperidone treatment produced a progressive across-session decline in avoidance responding to both CS1 and CS2. Olanzapine and risperidone dose-dependently disrupted the CS2 (a less salient conditioned stimulus) avoidance to a greater extent than the CS1 avoidance. These findings together with our previous ones, suggest that olanzapine and risperidone disrupt avoidance responding by attenuating the motivational salience of the conditioned stimulus (CS), while the baseline difference in the number of CS1 and CS2 avoidances is not a critical factor in determining their differential effects on CS1 and CS2 avoidances. In addition, we showed that prior risperidone treatment enhanced later avoidance response to olanzapine treatment, suggesting a possible shared interoceptive drug state and a cross-sensitization between these two drugs.

The present study was primarily designed to differentiate two alternative explanations of the preferential disruptive effect of antipsychotics on CS2 over CS1 avoidance responses. One explanation ("motivational salience attenuation") suggests that the greater disruption on CS2 avoidance is due to the weaker motivational salience of the CS2 relative to the CS1 (i.e. the ability to elicit avoidance). Another explanation ("different baseline response") suggests that it is due to the lower number of CS2 avoidance than CS1 avoidance at the baseline (predrug). The second explanation is consistent with a large number of reports showing that the response rate is an important determinant of the behavioral effects of drugs. This phenomenon is best known as the "rate-dependent drug effects" (Barrett et al., 2008; Leander, 1975; Leander and McMillan, 1974). For example, it has been shown that antipsychotic drugs (e.g. haloperidol, clozapine, and chlorpromazine), anxiolytic drugs (e.g. chlordiazepoxide), and other psychoactive drugs (e.g. 
amphetamine) can decrease or increase a behavioral response (e.g. lever-pressing, pecking, nose-poking) depending on the specific schedules of reinforcement (a fixed-interval schedule or a multiple schedule with alternating fixed-ratio and fixedinterval components) (Barrett, 1983; Harris et al., 1978; Spealman et al., 1983). Our previous study could not distinguish between these two explanations because not only was the CS2 made to be less salient than the CS1, but also the rate of CS2 avoidance was lower than the CS1 avoidance (Li et al., 2009b). Thus, both explanations could account for the preferential disruptive effect of antipsychotics on CS2 over CS1 avoidance responses. In the present study, we attempted to separate these two factors. As we did in our previous study, we rendered the CS2 less salient than the CS1 by pairing the CS1 with the US in every CS1 trial (100\%) while pairing the CS2 with the US in only $75 \%$ of the CS2 trials. Different from our previous study, we used more CS2 trials (i.e. 20) than CS1 trials (i.e. 10) in each training/testing session, making the rate of the CS2 avoidance (e.g. mean $=14$ on the pre-drug day) higher than that of the CS1 avoidance (e.g. mean $=9$ on the pre-drug day) (see Figure 1). Under this new condition, we still found that olanzapine and risperidone preferentially disrupted the CS2 avoidance over the CS1 avoidance. Collectively, findings from both studies indicate that the baseline rate of avoidance responses does not seem to influence the basic avoidance-disruptive effect of olanzapine and risperidone, which is in agreement with other reports in the literature suggesting that the effects of some antipsychotic drugs, such as thioridazine, chlorpromazine and haloperidol can be largely independent of the type of schedule or the type of consequent event that maintains response (Spealman et al., 1983; Wenger, 1979). Therefore, our findings supported the "motivational salience attenuation" explanation and refuted the "different baseline response" explanation. This idea is also supported by our finding that haloperidol-treated rats tested under a 40-trial avoidance testing session showed a faster decline than those tested under a 10-trial session, which in turn, showed a faster decline than those tested under a 3-trial session (Li et al., 2007). These findings, together with our previous ones (Li et al., 2009b), are also consistent with clinical observations that a less salient psychotic thought or hallucination is easier to be corrected and more responsive to drug treatment than a more persistent psychotic symptom (Manschreck and Khan, 2006).

As we have previously discussed (Li et al., 2009b), this idea that antipsychotics attenuate motivational salience of stimuli (e.g. decrease incentive motivation of rats) is consistent with the motivational salience theory of dopamine (Beninger, 2006; Berridge, 2007; Berridge and Robinson, 1998). According to this theory, dopamine is centrally involved in attributing incentive salience to objects or behavioral acts. This attributed salience then facilitates approach (to positive reinforcers) or avoidance (to negative reinforcers) responses. It has been shown previously that the effects of antipsychotics in the CAR model are dependent upon $\mathrm{D}_{2}$ blockade in the nucleus accumbens ( $\mathrm{Li}$ et al., 2010; Wadenberg et al., 1990). Therefore, the common behavioral mechanism shared by haloperidol, olanzapine, and risperidone may be attributed to their common antagonistic action on the $\mathrm{D}_{2}$ receptor. Accordingly, antagonism of $\mathrm{D}_{2}$ receptor function by haloperidol, olanzapine, or risperidone may inhibit the actions elicited by the CS and inhibit a weaker stimulus-elicited behavior to a greater extent than a stronger one (Ikemoto and Panksepp, 1999; Salamone and Correa, 2002).

The secondary aim of the present study was to examine the interoceptive drug state mechanism, which is hypothesized to play a role in maintaining the avoidance-disruptive effect across sessions and long-term antipsychotic drug sensitization (Li et al., 2007, 2009a; Mead and Li, 2010). This interoceptive drug state mechanism has been revealed in several ways in the past. In Mead and Li (2010), we reported that haloperidol and olanzapine on an intermittent (on-off-on) drug treatment regimen still produced a progressively enhanced disruption on avoidance responding as we observed in rats tested in the consecutive schedule ( $\mathrm{Li}$ et al., 2007), despite the fact that they all exhibited a high level of avoidance immediately prior to each drug session (e.g. during the drug-free re-training sessions). We also found that rats previously treated with olanzapine $(0.5-2.0 \mathrm{mg} / \mathrm{kg}$, sc) or risperidone $(0.2-1.0 \mathrm{mg} / \mathrm{kg})$ during the acquisition phase of avoidance conditioning exhibited significantly fewer avoidance responses when they were retested 3 weeks later to the same drug in comparison to rats that were previously treated with non-antipsychotic drugs (chlordiazepoxide, $10 \mathrm{mg} / \mathrm{kg}$, citalopram $10 \mathrm{mg} / \mathrm{kg}$, or sterile water). In $\mathrm{Li}$ et al. (2009a), we reported that pairing chlordiazepoxide (a cueing drug CS) with haloperidol engendered chlordiazepoxide to exhibit an acquired haloperidol-like property in disrupting avoidance responding. We also showed that rats previously treated with haloperidol showed a stronger response to the avoidance-disruptive effect of olanzapine than drug-naïve rats (Li et al., 2007). Similarly, rats previously treated with olanzapine showed a stronger response to the avoidance-disruptive effect of haloperidol than drug-naïve rats (Mead and $\mathrm{Li}$, 2010), suggesting that the antipsychotic drug state induced by haloperidol and olanzapine share some similarities. The present study examined the possible shared interoceptive drug state between olanzapine and risperidone. We found an asymmetric feature between olanzapine and risperidone: rats previously treated with risperidone $(1.0 \mathrm{mg} / \mathrm{kg})$ showed a stronger response to the avoidance-disruptive effect of olanzapine, whereas rats previously treated with olanzapine $(0.5$ and $1.0 \mathrm{mg} / \mathrm{kg}$ ) showed no such enhanced response to risperidone. One possible reason why this happened is that risperidone $1.0 \mathrm{mg} / \mathrm{kg}$ caused a much stronger disruption than olanzapine $1.0 \mathrm{mg} / \mathrm{kg}$ (see Figures 2 \& 3), thus risperidone may have caused a stronger sensitization-like effect than olanzapine. Were a higher dose of olanzapine (e.g. $2.0 \mathrm{mg} / \mathrm{kg}$ ) used, we may also be able to observe an enhanced response (i.e. lowered avoidance) to the challenge risperidone. Future work should employ a broader range of olanzapine and risperidone doses to further examine whether these drugs do share a common antipsychotic interoceptive drug state and whether prior experience with one drug can be transferred to another drug. This issue is of clinical importance because if there is such a shared interoceptive drug state and bidirectional exchange of drug sensitization between olanzapine and risperidone, clinicians working with patients who wish to switch from one drug (e.g. olanzapine ) to another drug (e.g. risperidone) may not need to be concerned with the possible change in antipsychotic efficacy during this process. However, if there is only one directional exchange of drug experience (e.g. from risperidone to olanzapine), clinicians may need to monitor patients' symptom response to the new drug during this switching process.

Taken together, the present study used a novel avoidance conditioning model and showed that atypical antipsychotics olanzapine and risperidone disrupt avoidance responding elicited by a weak stimulus to a greater extent and at a faster rate than one elicited by a strong stimulus, and prior antipsychotic experience may be maintained over time through an interoceptive drug state mechanism. To carefully extrapolate to clinical treatment, antipsychotic drugs may achieve their anti"psychotic" effect via a dual action: (a) selectively weakening the aberrant motivational salience of stimuli (e.g., psychotic thoughts or abnormal perceptions, internal and external cues); and (b) producing a drug interoceptive state that allows the weakening effect on the motivational salience of stimuli to be maintained over time. 
Acknowledgments - This study was funded in part by the NIMH grant (R01MH085635) to Professor Ming Li. We thank Ms. Natashia Swalve, Ms. Heidi Gonzalez, and Mr. Nick Volf for their editorial help.

\section{References}

Arnt J. Pharmacological specificity of conditioned avoidance response inhibition in rats: inhibition by neuroleptics and correlation to dopamine receptor blockade. Acta Pharmacol Toxicol (Copenh) 1982; 51:321-9.

Barrett JE. Comparison of the effects of antipsychotic drugs on the schedule-controlled behavior of squirrel monkeys and pigeons. Neuropharmacology 1983; 22:519-24.

Barrett JE. The emergence of behavioral pharmacology. Mol Interv 2002; 2:470-5.

Barrett JE, Bergman J, Peter B. Dews and pharmacological studies on behavior. J Pharmacol Exp Ther 2008; 326:683-90.

Beninger RJ. Dopamine and incentive learning: a framework for considering antipsychotic medication effects. Neurotox Res 2006; 10:199-209.

Berridge $\mathrm{KC}$. The debate over dopamine's role in reward: the case for incentive salience. Psychopharmacology (Berl) 2007; 191:391-431.

Berridge KC, Robinson TE. What is the role of dopamine in reward: hedonic impact, reward learning, or incentive salience? Brain Res Brain Res Rev 1998; 28:309-69.

Bignami G. Effects of neuroleptics, ethanol, hypnotic-sedatives, tranquilizers, narcotics, and minor stimulants in aversive paradigms. In: Anisman H, Bignami G, editors. Psychopharmacology of aversively motivated behavior. New York and London: Plenum Press; 1978. p. 385-453.

Cook L, Davidson AB. Behavioral pharmacology: animal models invovling aversive control of behavior. In: Lipton MA, DiMascio A, Killam K, editors. Psychopharmacology: a generation of progress. New York: Raven; 1978. p. 563-7.

Dews PB. Interactions of behavioral effects of drugs. Ann NY Acad Sci 1976; 281:50-63.

Harris RA, Snell D, Loh HH. Effects of D-amphetamine, monomethoxyamphetamines and hallucinogens on schedule-controlled behavior. J Pharmacol Exp Ther 1978; 204:103-17.

Ikemoto S, Panksepp J. The role of nucleus accumbens dopamine in motivated behavior: a unifying interpretation with special reference to reward-seeking. Brain Res Brain Res Rev 1999; 31:6-41.

Janssen PAJ, Niemegeers CJE, Schellekens KHL. Is it possible to predict the clinical effects of neuroleptic drugs (major tranquilizers) from animal data? Arzneimittelforschung 1965; 15:104-17.

Kapur S, VanderSpek SC, Brownlee BA, Nobrega JN. Antipsychotic dosing in preclinical models is often unrepresentative of the clinical condition: a suggested solution based on in vivo occupancy. J Pharmacol Exp Ther 2003; 305:625-31.

Kuribara H, Tadokoro S. Correlation between antiavoidance activities of antipsychotic drugs in rats and daily clinical doses. Pharmacol Biochem Behav 1981; 14:181-92.

Leander JD. Rate-dependent Effects of drugs. II. Effects of some major tranquilizers on multiple fixed-ratio, fixed-interval schedule performance. J Pharmacol Exp Ther 1975; 193:689-700.
Leander JD, McMillanDE. Rate-dependent effects of drugs. I. Comparisons of D-amphetamine, pentobarbital and chlorpromazine on multiple and mixed schedules. J Pharmacol Exp Ther 1974; 188:726-39.

Li M, Fletcher PJ, Kapur S. Time course of the antipsychotic effect and the underlying behavioral mechanisms. Neuropsychopharmacology 2007; 32:263-72.

LiM,He W,Mead A.An investigation of the behavioralmechanisms of antipsychotic action using a drug-drug conditioning paradigm. Behav Pharmacol 2009a; 20:184-94.

Li M, He W, Mead A. Olanzapine and risperidone disrupt conditioned avoidance responding in phencyclidine-pretreated or amphetamine-pretreated rats by selectively weakening motivational salience of conditioned stimulus. Behav Pharmacol 2009b; 20:84-98.

Li M, Parkes J, Fletcher PJ, Kapur S. Evaluation of the motor initiation hypothesis of APD-induced conditioned avoidance decreases. Pharmacol Biochem Behav 2004; 78:811-9.

Li M, Sun T, Zhang C, Hu G. Distinct neural mechanisms underlying acute and repeated administration of antipsychotic drugs in rat avoidance conditioning. Psychopharmacology (Berl) 2010; 212:45-57.

Manschreck TC, Khan NL. Recent advances in the treatment of delusional disorder. Can J Psychiatry 2006; 51:114-9.

McMillan DE, Katz JL. Continuing implications of the early evidence against the drivereduction hypothesis of the behavioral effects of drugs. Psychopharmacology (Berl) 2002; 163:251-64.

Mead A, Li M. Avoidance-suppressing effect of antipsychotic drugs is progressively potentiated after repeated administration: an interoceptive drug state mechanism. J Psychopharmacol 2010; 24:1045-53.

Salamone JD, Correa M. Motivational views of reinforcement: implications for understanding the behavioral functions of nucleus accumbens dopamine. Behav Brain Res 2002; 137:3-25.

Shannon HE, Hart JC, Bymaster FP, Calligaro DO, DeLapp $\mathrm{NW}$, Mitch $\mathrm{CH}$, et al. Muscarinic receptor agonists, like dopamine receptor antagonist antipsychotics, inhibit conditioned avoidance response in rats. J Pharmacol Exp Ther 1999; 290:901-7.

Spealman RD, Kelleher RT, Goldberg SR, DeWeese J, Goldberg DM. Behavioral effects of clozapine: comparison with thioridazine, chlorpromazine, haloperidol and chlordiazepoxide in squirrel monkeys. J Pharmacol Exp Ther 1983; 224:127-34.

van der Heyden JA, Bradford LD. A rapidly acquired oneway conditioned avoidance procedure in rats as a primary screening test for antipsychotics: influence of shock intensity on avoidance performance. Behav Brain Res 1988; 31:61-7.

Wadenberg ML, Ericson E, Magnusson O, Ahlenius S. Suppression of conditioned avoidance behavior by the local application of (-)sulpiride into the ventral, but not the dorsal, striatum of the rat. Biol Psychiatry 1990; 28:297-307.

Wadenberg ML, Hicks PB. The conditioned avoidance response test re-evaluated: is it a sensitive test for the detection of potentially atypical antipsychotics? Neurosci Biobehav Rev 1999; 23:851-62.

Wenger GR. Effects of clozapine, chlorpromazine and haloperidol on schedule-controlled behavior. Pharmacol Biochem Behav 1979; 11:661-7. 